\title{
Nasal Anatomy and Analysis
}

\section{Guy Kenyon}

\section{ABSTRACT}

This article describes the anatomy of the nose and the principles of analysis of the nose and face that will aid a successful rhinoplasty. The analysis is based on the Caucasian female face and appropriate alterations will be needed in considering male patients and those with a differing ethnicity.

Keywords: Nose, Surgery, Rhinoplasty, Anatomy, Analysis.

How to cite this article: Kenyon G. Nasal Anatomy and Analysis. Int J Otorhinolaryngol Clin 2013;5(1):34-42.

Source of support: Nil

Conflict of interest: None declared

\section{INTRODUCTION}

Rhinoplasty is an operation where millimeters of change can have a marked effect on outcome. It is therefore imperative that the operating surgeon has a good understanding of the anatomy of the nose and surrounding structures and of their inherent variations. ${ }^{1}$

The nose is made of three basic components: framework, support and external cover. The nasal framework consists of the skeletal framework of cartilages and bones. The support is provided by tissues and ligaments that hold the intrinsic framework together, and the skin and soft tissues form the external cover. These components are intricately related and must be born in mind in every step of a septorhinoplasty.

The principles that must be adhered to in rhinoplasty consist of:

1. A precise definition of the abnormality or abnormalities present and the cause of these abnormalities, as far as can be ascertained from preoperative examination.

2. Definition of the goals to be achieved.

3. Adequate exposure of the nasal deformity.

4. Preservation or restoration of normal anatomy.

5. Incremental and planned correction of the specific abnormalities identified.

6. Maintenance or restoration of the nasal airway.

\section{SKIN}

The skin of the nose is thinner and more mobile in the upper two thirds of the nose and is thicker and more adherent in the distal third. In the middle third, over the keystone area, the skin is at its thinnest (Fig. 1).

It is important to note the type, texture and sebaceous content of the skin as it will have an effect upon the final

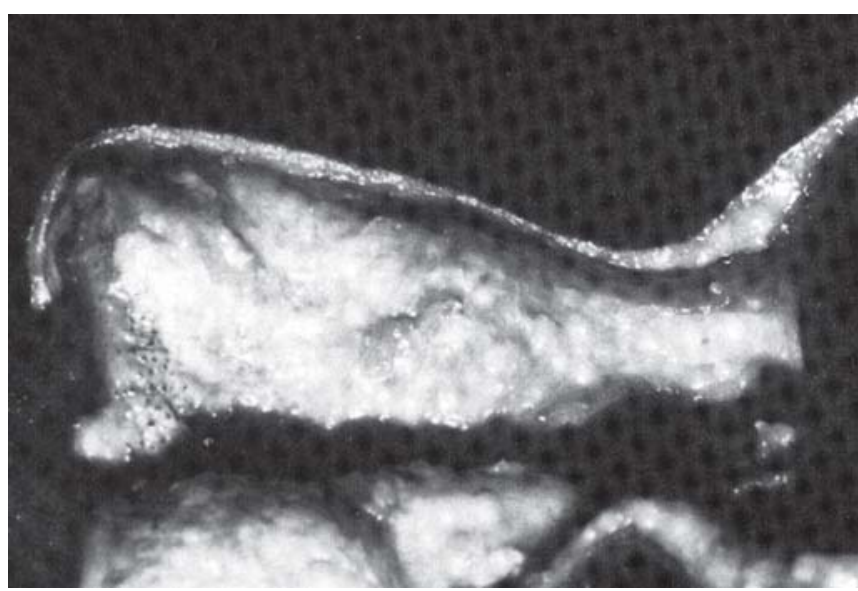

Fig. 1: The side of the nose showing thickness of the nasal skin

result. For example, in a Caucasian female with thin skin any alteration of the underlying framework with a resultant minor irregularity will likely show after time and provide an adverse outcome. By contrast a patient with thick seborrheic skin may require a more aggressive alteration of the underlying framework to achieve the desired outcome.

\section{MUSCLES}

The vessels and nerves pass within the subcutaneous tissues above the muscles. There are several muscles but their exact disposition is a matter of debate and one study showed not agreement as to their exact form (Figs 2A to C). ${ }^{2}$ However, this, in practice, matters little as only two have any clinical significance (Fig. 3).

1. The levator labii alaeque nasi assists in keeping the external valve open and assists in alar flaring with the dilator nares. Nasal obstruction can occur if flaring is impeded and may be a result of facial paralysis in some patients.

2. The depressor septi nasi muscle. This originates from the orbicularis oris and runs onto the medial crura. This can shorten the upper lip and may, in some instances, result in decreased tip projection with animation.

\section{BLOOD SUPPLY}

There has been some concern in the past, especially with the tendency to open approach rhinoplasty, that the transcolumellar and other incisions may compromise the blood supply to the nasal tip.

The blood supply is, in fact, quite rich (Figs 4 and 5). The anterior ethmoids, dorsal nasal and external nasal arteries supply the proximal part of the nose and the nasal 

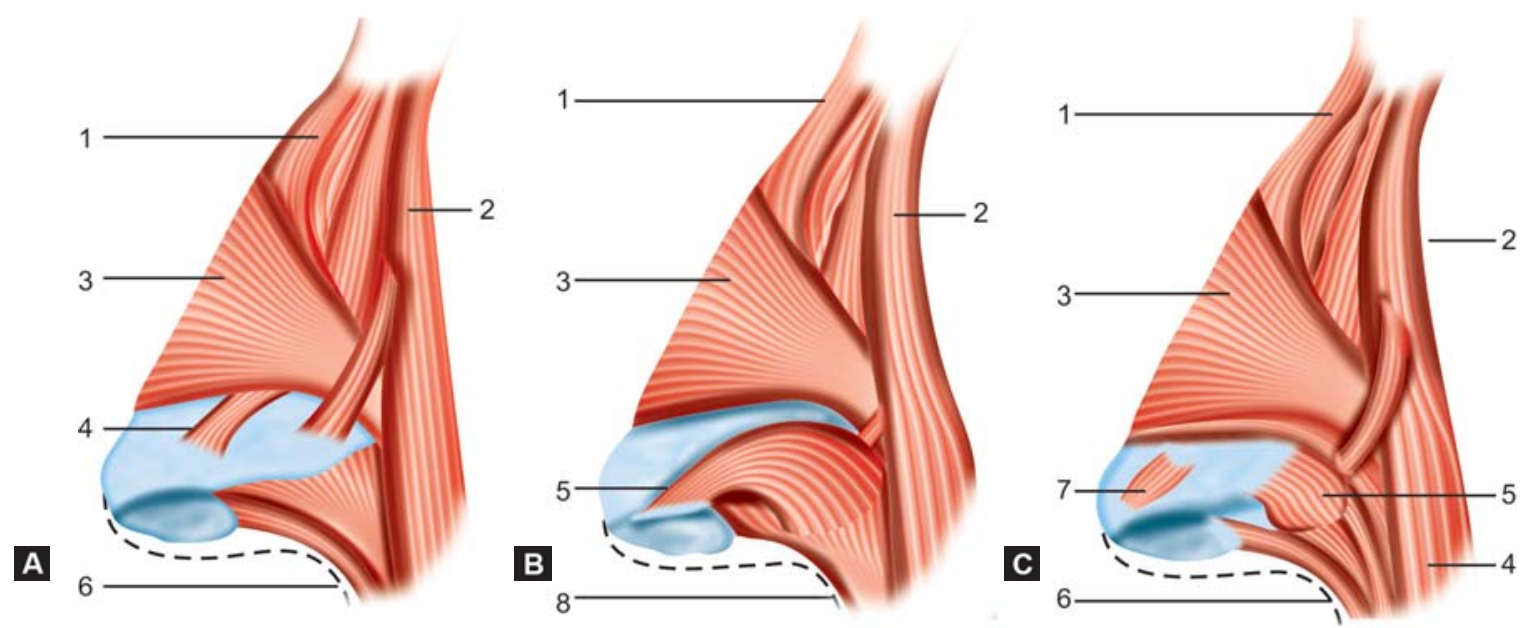

Figs 2A to C: Differing arrangements of the nasal muscles in differing anatomical texts ${ }^{2}$

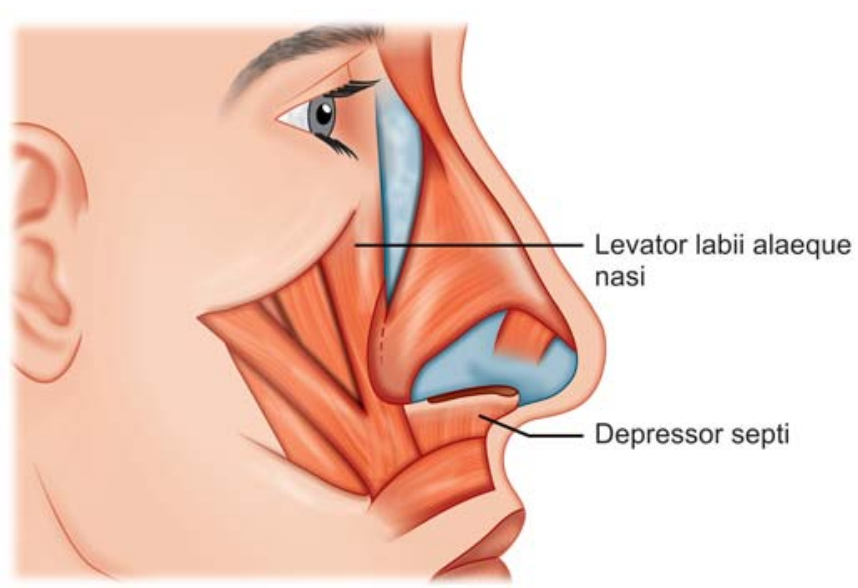

Fig. 3: Lateral view of the nose showing the nasal muscles of clinical importance

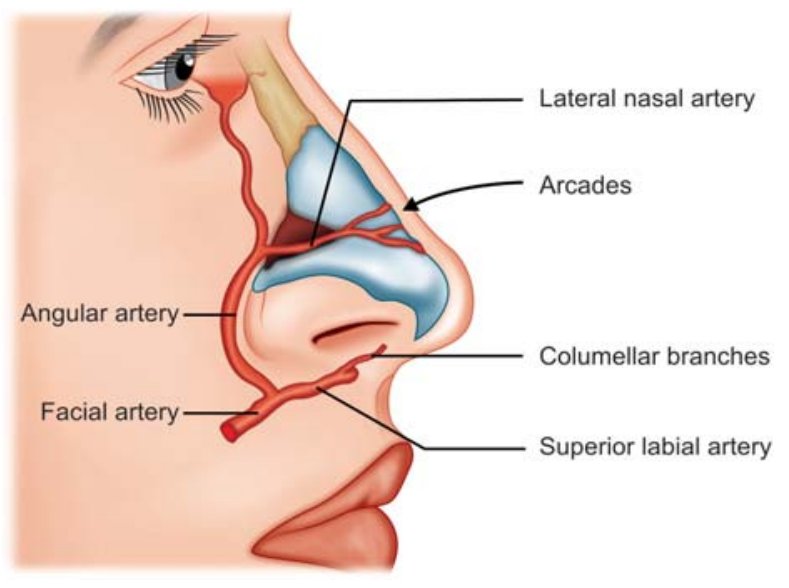

Fig. 4: Lateral view of the nose showing the principle arterial supply

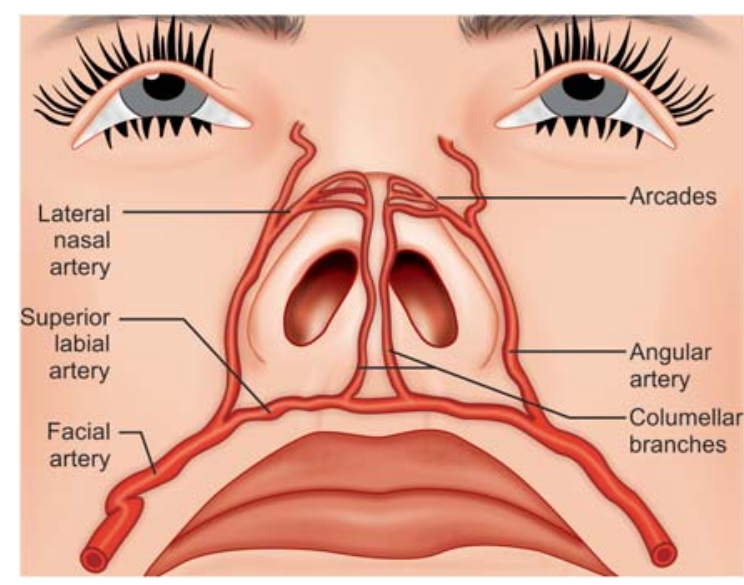

Fig. 5: Tip of the nose showing the arterial supply

\section{Bony Vault}

This is comprised of paired nasal bones as well as the ascending frontal process of the maxilla and this vault makes up the most cephalad third or half of the nose. The bones are narrowest above the intercanthal level but have increased 


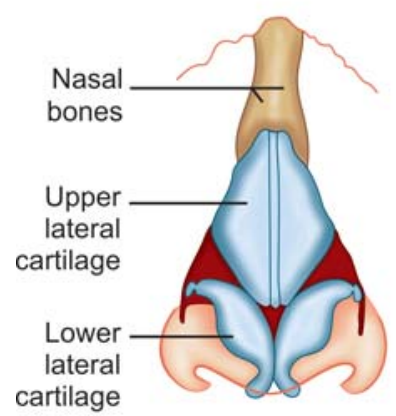

Anatomy

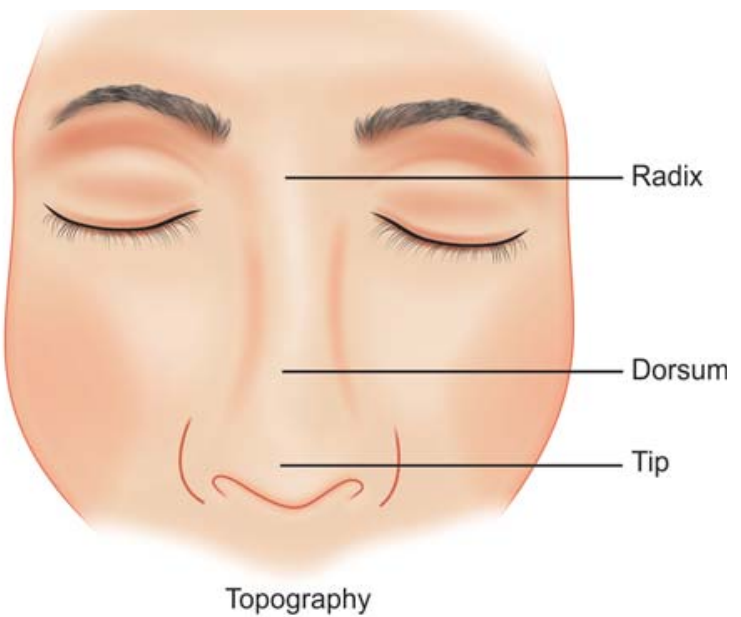

Topography

Fig. 6: The primary anatomy of the nasal skeleton and the clinically important landmarks (frontal view)
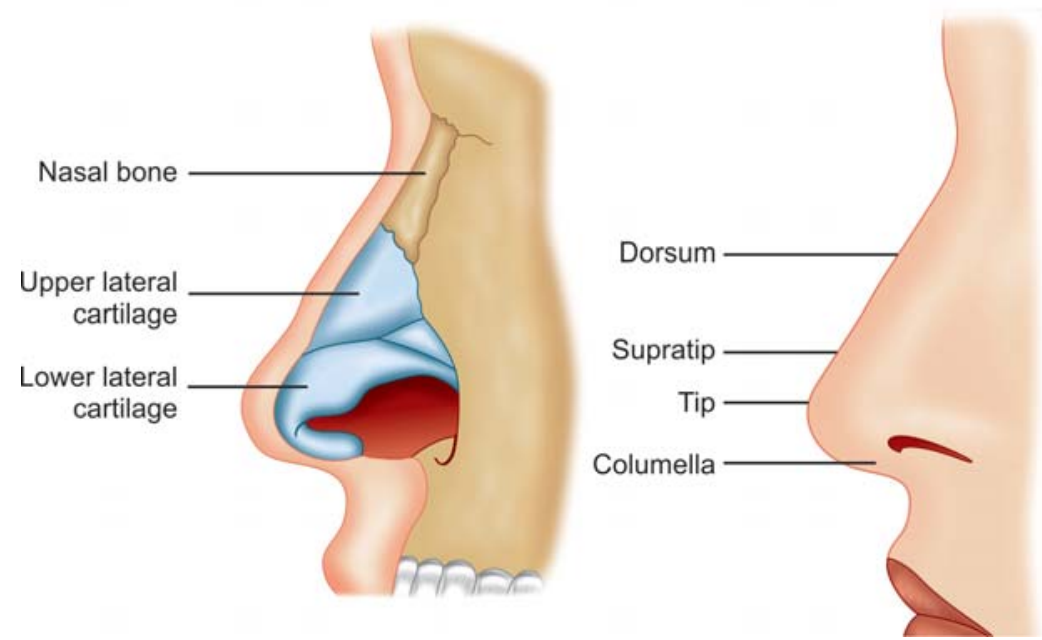

Fig. 7: The primary anatomy of the nasal skeleton and the clinically important landmarks (lateral view)

thickness in that area becoming progressively thinner toward the keystone area (Figs 8A and B). The clinical correlation of this is that osteotomies may prove difficult above the intercanthal line and are less unpredictable superiorly.

\section{Upper Cartilaginous Vault}

The upper lateral cartilages underlay the nasal bones for 6 to $8 \mathrm{~mm}$ and their junction form a tight synchondrosis. They extend down to the scroll area, which has variable
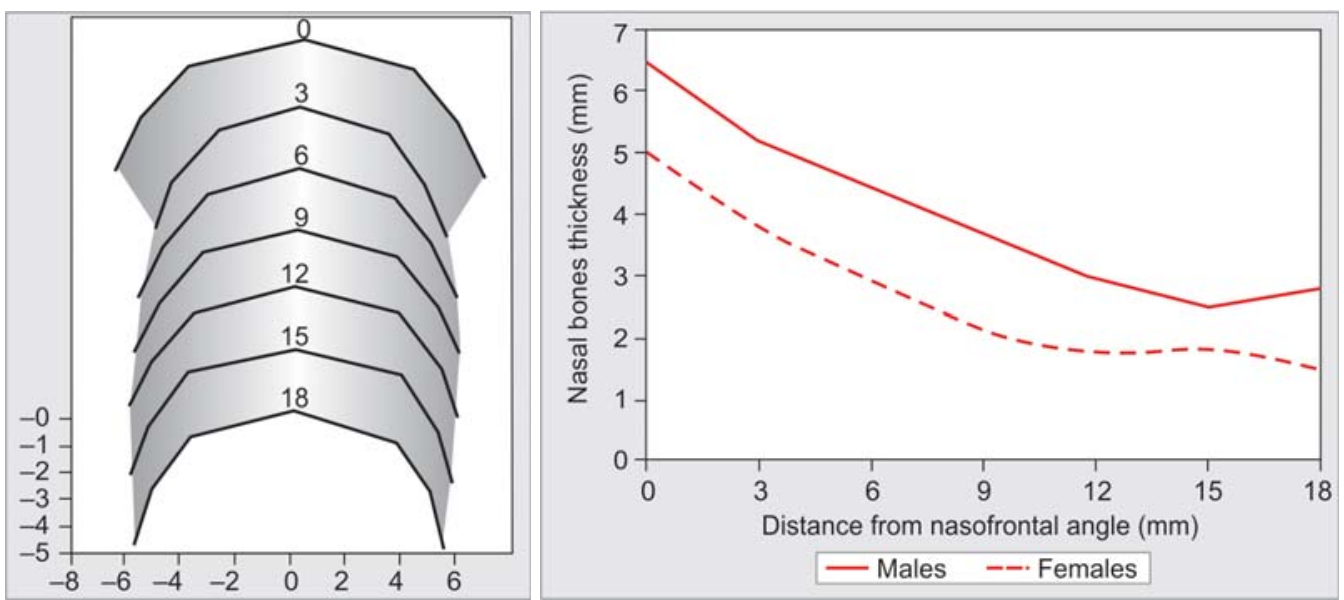

Fig. 8: Results from measuring bone thickness at different levels from the nasion of the nose (male and female) 
anatomy and which lies at the junction between the upper and lower lateral cartilages. The keystone area is the area formed by the junction of the upper lateral with the nasal bones and the dorsal septum. Internally this forms a T-shaped structure. The contour of the nasal dorsum is such that this area is the widest along the normal nasal dorsum and this must be born in mind when reconstruction is required.

\section{Lower Cartilaginous Vault}

The lowest vault is comprised of the medial, intermediate and lateral crura of the lower lateral cartilages (Fig. 9). These structures are important in normal tip projection and in maintenance of a normal nasal airway. They are supported by (Fig. 10):

1. The length and strength of the lower lateral cartilages.

2. The suspensory ligament of the tip.

3. The fibrous connections between the lateral crura and the caudal border of the upper lateral cartilages.

4. The abutment with the bony pyriform aperture.

5. The anterior septal angle.

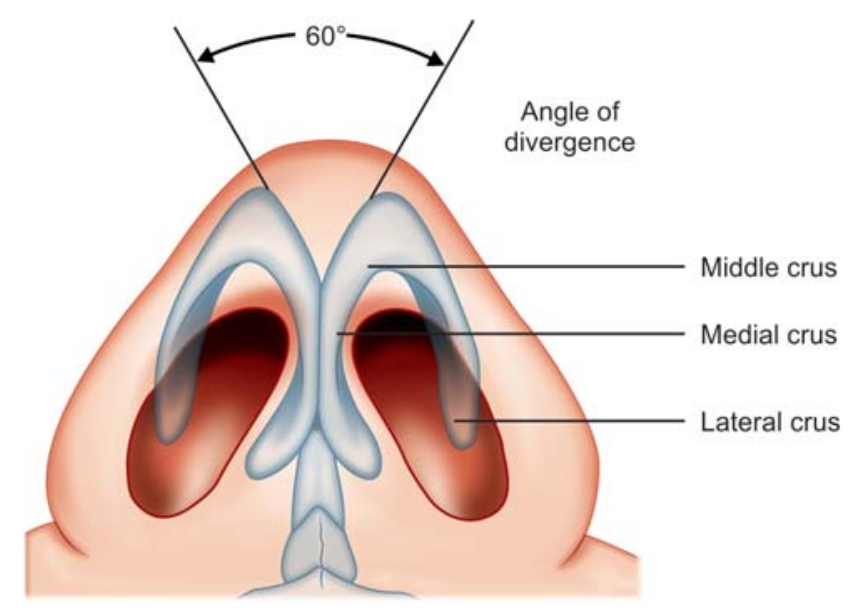

Fig. 9: The nasal tip and the arrangement of the lower lateral cartilages

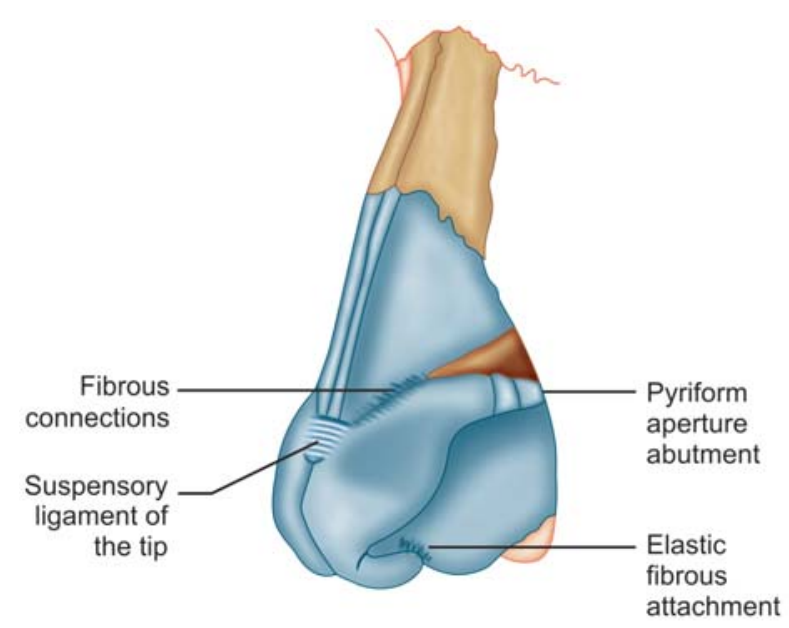

Fig. 10: The support mechanisms of the nasal tip
The angle of divergence of the lower lateral occurs at the middle crus (Fig. 9) and is an important factor in determining tip type - specifically in resulting in a boxy or bifid tip. The domes of the lower lateral form the tip defining points which, on photographs show as two highlighted areas in the overlying skin. In Caucasian patients two separate and distinct points are desirable on frontal view, whereas in the oriental nose a single tip defining point and a more amorphous look is frequently regarded as being more appealing.

\section{Nasal Airway}

The nasal septum divides the nose into two chambers. This structure is formed of the perpendicular plate of the ethmoid bone posteriorly which is in continuity with the cribriform plate above (Fig. 11). Undue and injudicious force in resecting posterior deviations can thus imperil the patient and expose him or her to the possibility of a cerebrospinal fluid (CSF) leak.

The most projecting part of the premaxilla is the nasal spine which should be preserved in some form to maintain an attachment to the most caudal part of the quadrilateral cartilage and to stabilize the nose during septal reconstruction. ${ }^{4}$ The quadrilateral cartilage joins posteriorly with the perpendicular plate of the ethmoid and sits in a groove formed by the maxilla inferiorly. Posteriorly these structures articulate with the vomer (Fig. 11).

Internally the junction of the upper lateral cartilage and the dorsal septum forms the internal nasal valve (Figs 12A and $\mathrm{B}$ ). This structure is somewhat misnamed since a valve regulates or controls the direction of flow: conspicuously the latter function is not a matter for the nose and any control of flow can only be passive and is dependant upon the mucosal activity in and around the head of the inferior turbinate. What is understood is that the angle between the upper lateral and the dorsal septum should be 10 to $15^{\circ}$ to allow for a perception of unrestricted airflow and

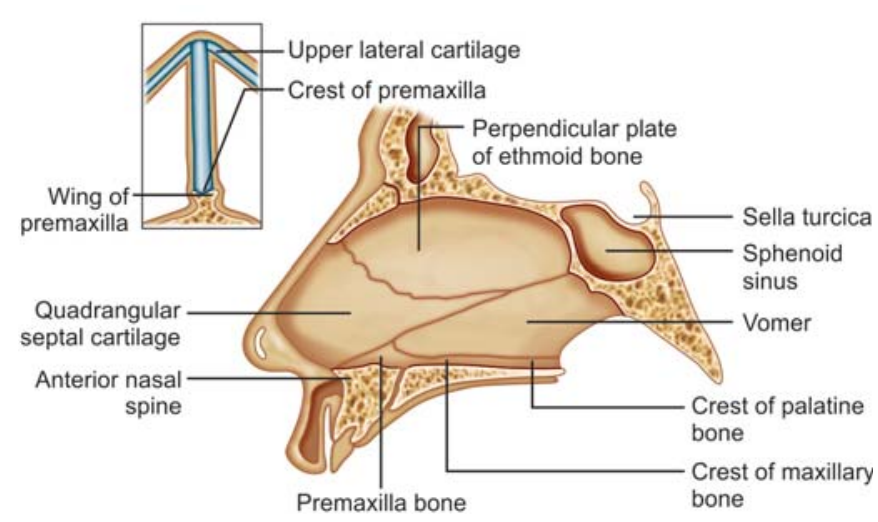

Fig. 11: The nasal septum and its connections to the upper lateral cartilages in the middle vault of the nose 

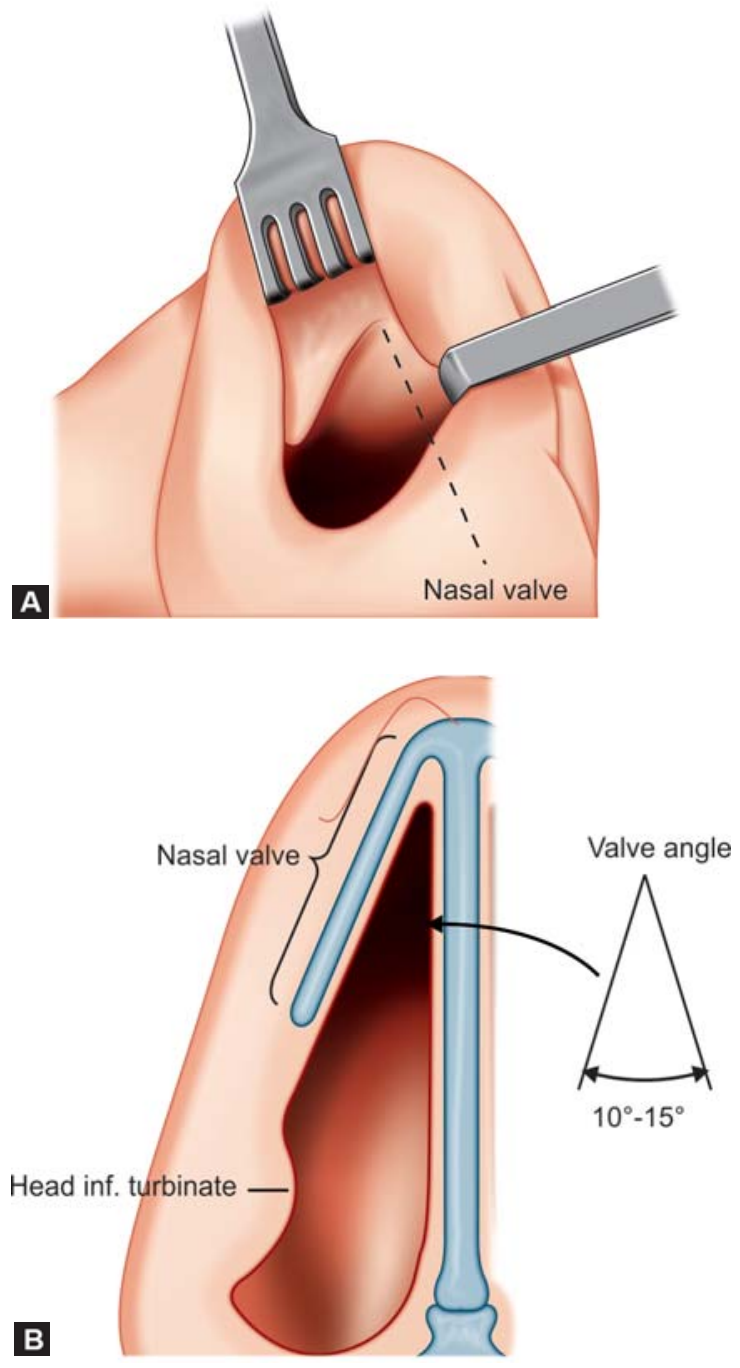

Figs $12 A$ and $B:$ The nasal valve

reconstruction in this area (Figs 12A and B) by means of a spreader graft may be required as part of a primary or secondary rhinoplasty to reconstruct and widen the nose in this coronal plane - especially if the bony vault is poorly formed and the middle third length is proportionally higher: Such circumstances predispose to internal valve deformity and narrowing.

There are three turbinate bones but the inferior is by far and away the most important in terms of nasal function. Out-fracture of this structure with either partial resection of the underlying bone (turbinoplasty) or reductions of the overlying mucosa (submucosal diathermy or coblation) are common accompaniments of intranasal airway surgery. The airway is narrowest in the internal valve area and the head of the inferior turbinate lies in this coronal plane. The airway is thus maximally corrected by surgery to the anterior part of this structure. More posteriorly the gain in airway patency from surgery is considerably less and brisk bleeding may also occur posteriorly.

\section{FACIAL ANALYSIS}

Attractive faces are held to have certain proportions and relationships in common. To make an accurate diagnosis and to formulate a treatment plan requires analysis both of the face and of the nose. Some features may only be correctable using orthognathic or even craniofacial approaches which the patient may not desire. However, such discrepancies should be noted and discussed especially since they may significantly affect the outcome for the patient. The following account relates to a Caucasian female and is an idealistic compilation of desired facial measurements and proportions. These relationships are not absolutes since there are many attractive faces that do not have these proportions. However, they do provide the basis for some form of understanding what features give a face its individual appearance and some insights as to how abnormalities may be corrected.

When beginning the normal examination it is important to notes first the skin type and texture. If it is thick and sebaceous it will not drape as well as thinner skin and it will take much longer for the edema associated with surgery to dissipate. Most sebaceous skin is thick but occasionally a patient will have thin oily skin. The thickness of the skin, rather than its seborrheic quality, is normally considered to be more important in predicting how it will drape.

Facial symmetry is next assessed. No face is entirely symmetrical and significant asymmetries, particularly a degree of mandibular disproportion, may not have been apparent to the patient previously. They should be pointed out tactfully during the initial consultation and noted in order to avoid the impression to the patient, after surgery, that they were somehow the result of the intervention performed.

It should be possible to divide the face into vertical onefifths (Fig. 13) and the distance between the brow to the menton should equal the width of the face at the malar level (Fig. 14). It should also be possible to divide the face into thirds by horizontal lines drawn adjacent to the menton, the brows (supraorbital ridges) and hairline. In turn the lower third can be divided by a line through the oral commissures into an upper one third and a lower two thirds (Fig. 15).

In these assessments the relationship between the mandible and the maxilla is important as is the degree of malar prominence. Because of the importance of lip position and contour in assessing tip projection and rotation the lip-chin complex should be evaluated before proceeding further with the nasal analysis. This requires a lateral view of the nose with the head in the natural horizontal facial plane (Fig. 16). If the upper lip is not in a good position and with a full and normal contour this detracts from the appearance. Some patients have a 'tension lip' which is characterized by fullness at the columella-labial angle and 


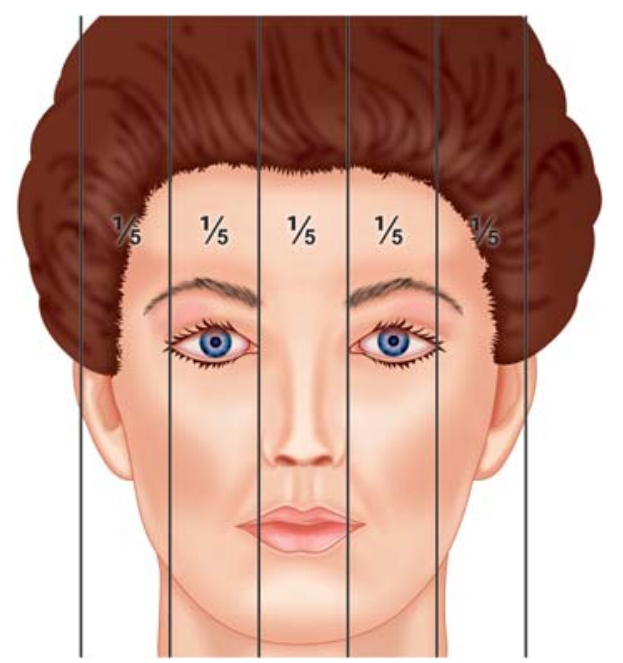

Fig. 13: Schematic diagram of the face and its principle subdivision into 5 ths

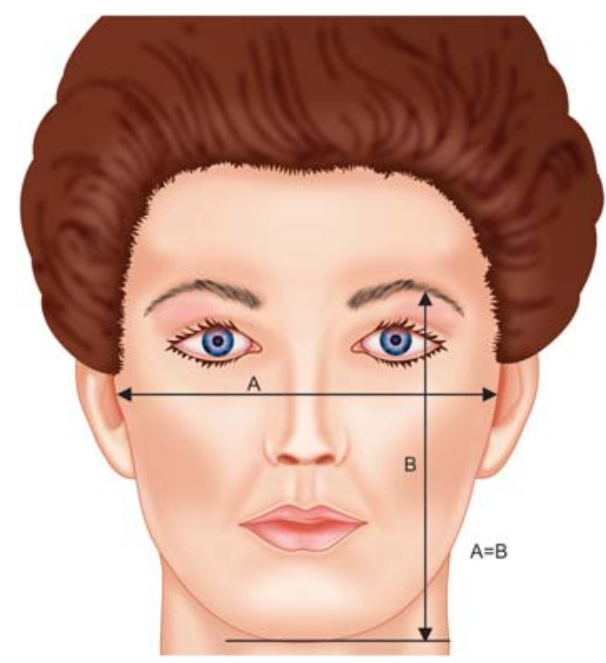

Fig. 14: Schematic diagram of the face and the relationship between mid facial width and the height of the face from eyebrow to menton

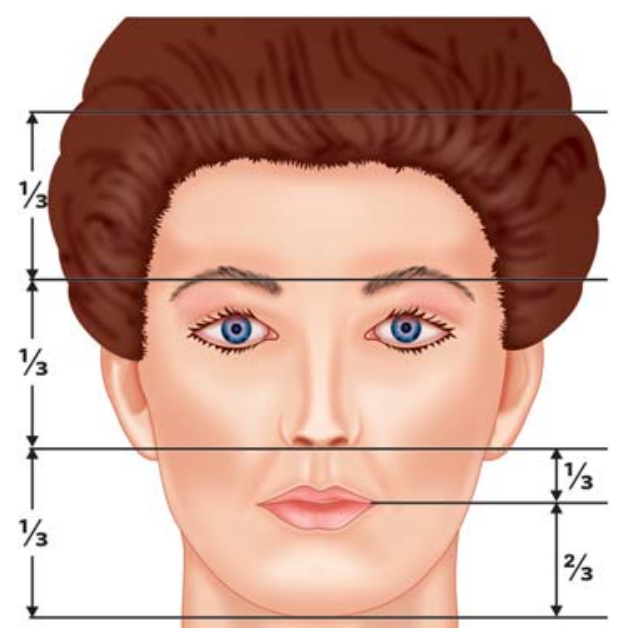

Fig. 15: Schematic diagram of nasal proportions in respect of the upper and lower thirds of the face

a flat lip with a thin vermillion surface that appears slightly retracted. This is most often seen in overprojected noses. A prominent caudal septum will also create abnormality with

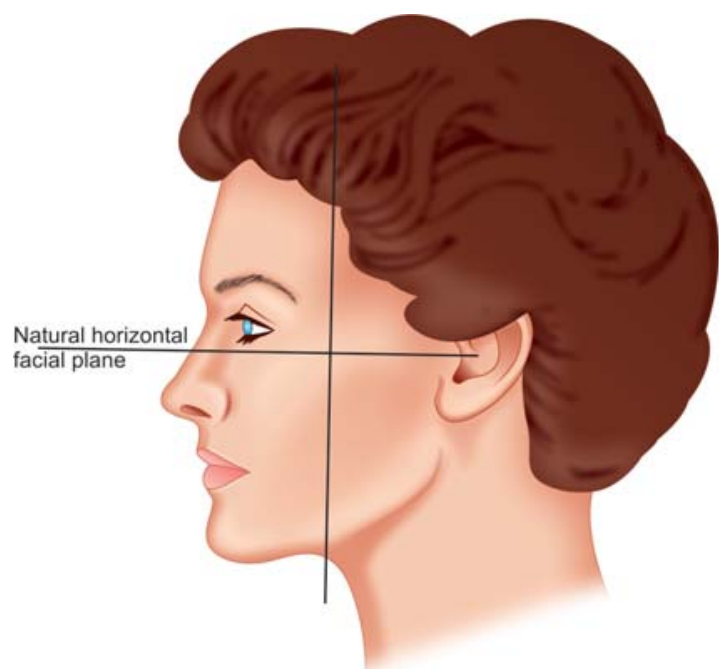

Fig. 16: The natural horizontal facial plane

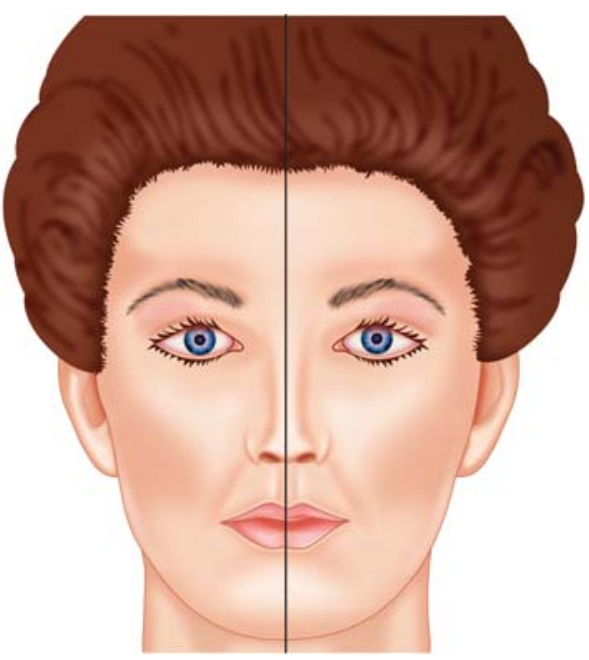

Fig. 17: The division of the face in assessing nasal symmetry

fullness at the columella-labial junction which gives the impression of a pseudorotation. Finally, prominent incisor teeth may also give increase projection of the upper lip.

After facial analysis is complete, nasal analysis is undertaken. The first parameter to assess is the degree of any deviation and its cause. Looking at the frontal view a line drawn from the midglabellar region to the menton should bisect the nasal bridge, nasal tip and the Cupid's bow (Fig. 17) and the dorsum should be outlined by two dorsal esthetic lines, running from the tip defining point to the medial aspect of the eyebrow on each side (Fig. 18). Next the width of the body and tip of the nose is assessed (Figs 19 and 20). The bony base should be $80 \%$ of the alar base width and the base width, in turn, should equal to the intercanthal distance or one eyes width.

If wider than this, consideration should be given to narrowing this at the time of osteotomy. If the base is correct, but the dorsum is wide, mobilization of the base is not appropriate and should be avoided. If it is wider than this, alar base resection should be considered. 


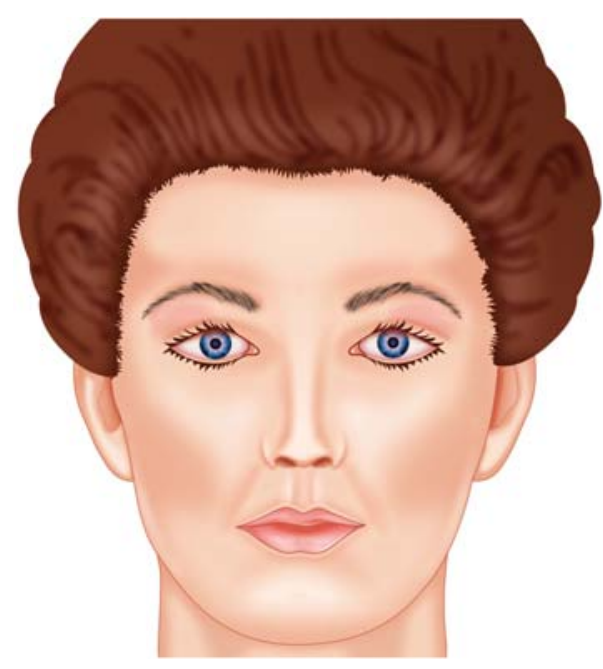

Fig. 18: The dorsal esthetic lines

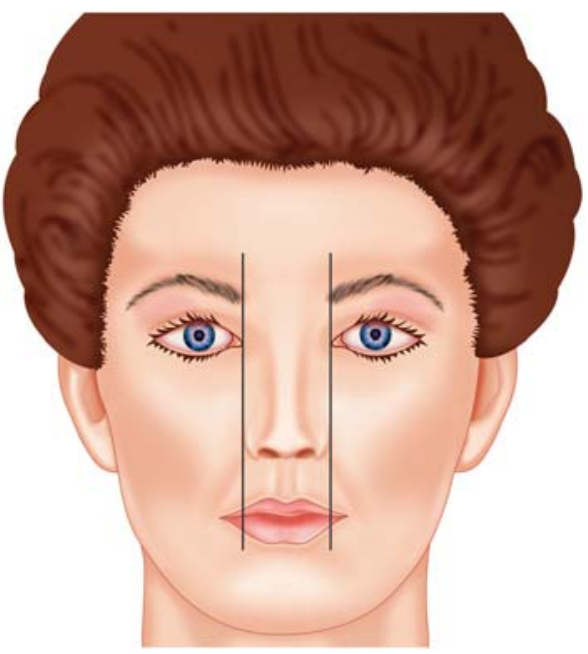

Fig. 19: The width of the nose and its relationship to the intercanthal distance

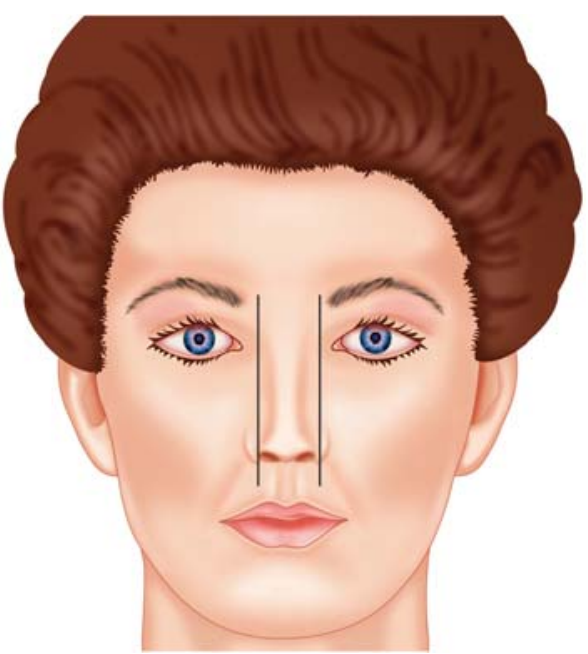

Fig. 20: The width of the nose in relation to the alar base width

The tip is next considered. Tip defining points are sought and lines connecting these, the supratip break and the columella lobular angle should form two equilateral triangles (Fig. 21). If any of these landmarks are out of position the

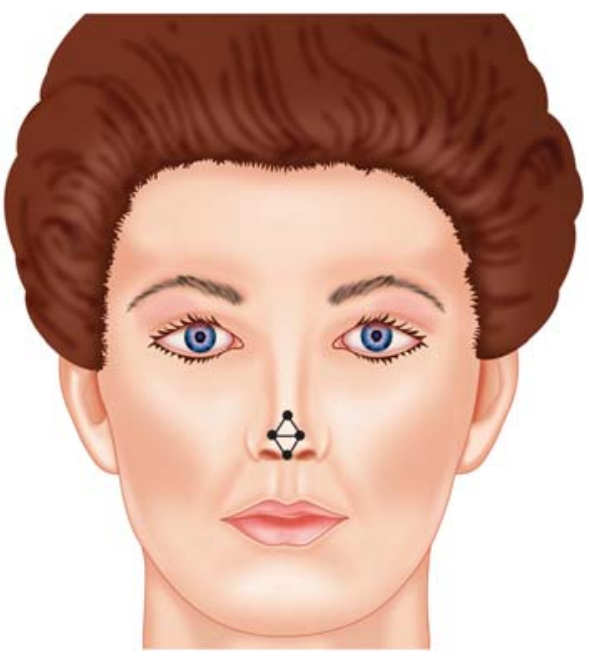

Fig. 21: The tip defining pints and their relationship to the supratip break and to the columella lobular angle

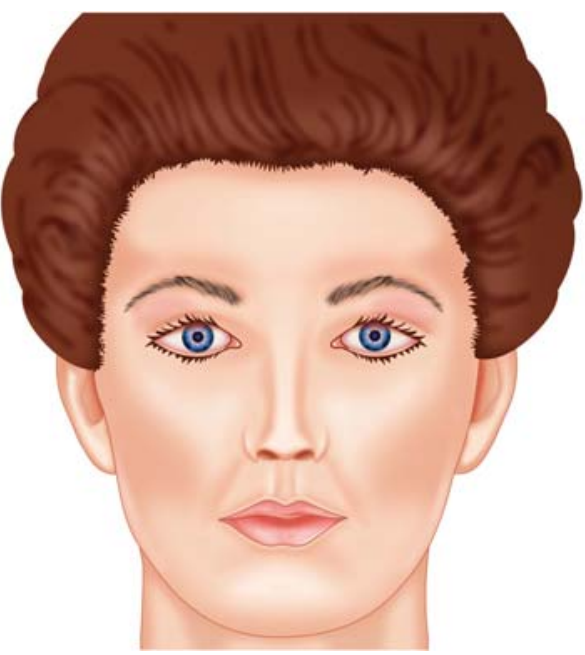

Fig. 22: The contour of the alar rims and nasal tip

cause should be identified and corrected at the time of surgery. The degree of bulbosity should also be assessed for, if the tip is bulbous, the lower lateral cartilages will have to be attenuated or sutures might have to be placed to reposition the domes. The columella is also assessed. In a normal nose it should hang just below the alar rims so that a line joining the rims and the lowest portion of the columella will give a gentle gull-wing appearance (Fig. 22). On inspection of the basal view (Fig. 23) an equilateral triangle should be visualized. The ratio of the columella to the lobular part of the nose should be 2:1 and the nostrils should be teardrop shaped with the long axis from the apex to the base orientated in a slightly lateral direction. The sides of the nostrils should be down to the point where the medial crura start to flare. If they flare too soon then the columella appears short and will detract from the tear dropped shape of the nostril. Resection of soft tissues between the crura and suturing them together will reduce flare.

With the eyes in forward gaze the nasofrontal angle should be at a level between the upper eyelashes and the 


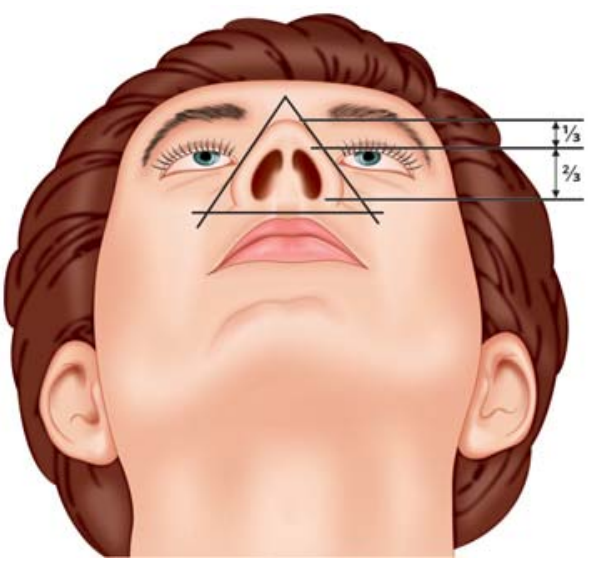

Fig. 23: Basal view showing the nasal proportions

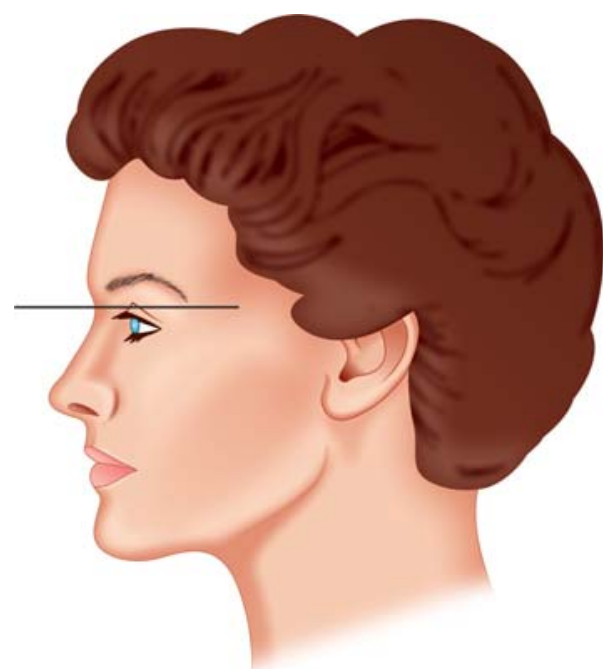

Fig. 24: The position of the nasofrontal angle

supratarsal creases (Fig. 24). This angle should be a gentle concave curve. Nasal tip projection is next assessed and there are various algorithms for this. Perhaps the simplest is to look at this is respect of the projection of the upper lip for if this is normal then 50 to $60 \%$ of the tip should lie anterior to a vertical line drawn adjacent to the most projected portion of the upper lip (Fig. 25). If over $60 \%$ lies anterior to this line, the tip is likely to be overprojected. If nasal length is correct then the ratio of nasal length to tip projection should be 1:0.67 (Fig. 26). Once the desired tip projection has been decided upon the nasal dorsum should then be assessed by drawing a line between the tip and the nasofrontal angle (Fig. 27). In women, the dorsum should lie approximately $2 \mathrm{~mm}$ behind this line with a small supratip break. In men the dorsum should be slightly higher.

Following this, the degree of tip rotation should be assessed. Rotation is determined by the nasolabial angle. If a straight line is drawn through the most anterior and posterior parts of the nostrils the angle is determined by measurement against a plumb line from the nasofrontal angle

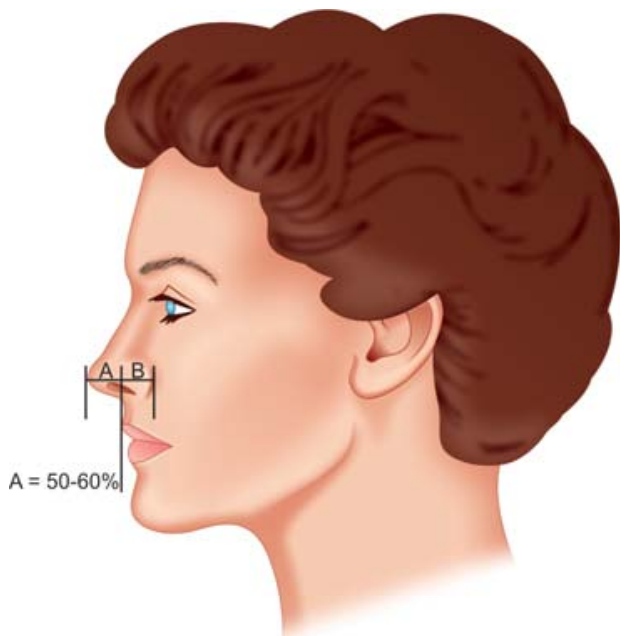

Fig. 25: Assessment of tip projection in relation to the upper lip

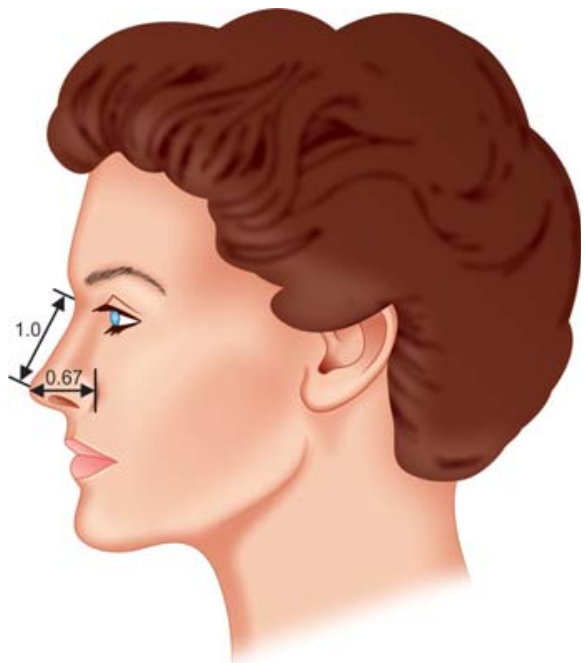

Fig. 26: Assessment of tip projection

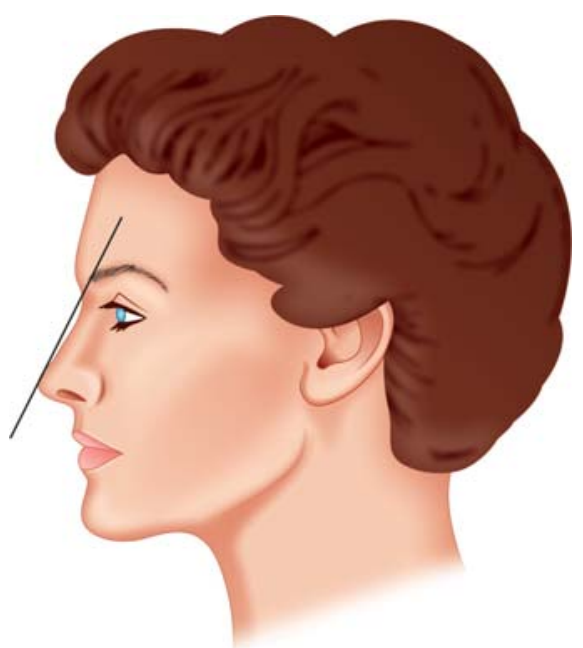

Fig. 27: Assessment of the position of the nasal dorsum

(Fig. 28). The angle should be 90 to $95^{\circ}$ in men and more than this is women $\left(95-100^{\circ}\right)$. The columella-labial angle is not the same and is the angle between the columella and the upper lip: this should be separately determined and, if 


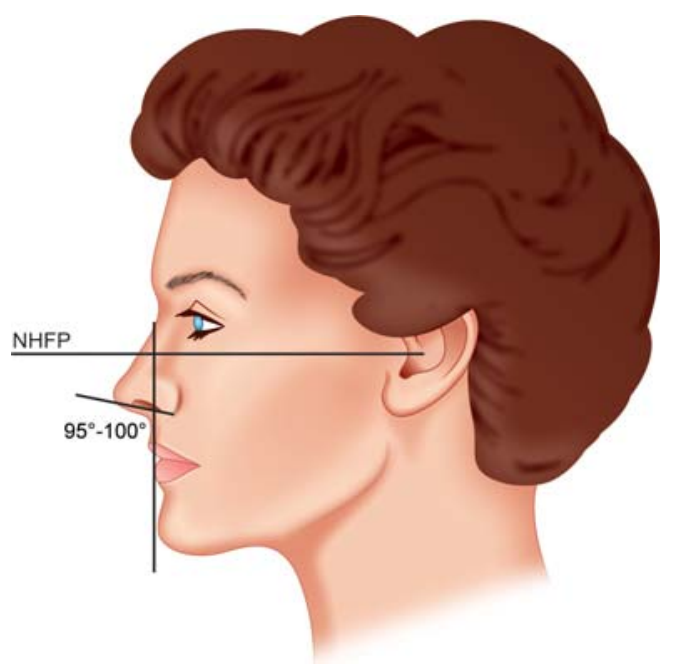

Fig. 28: Assessment of tip rotation in respect of the natural horizontal facial plane

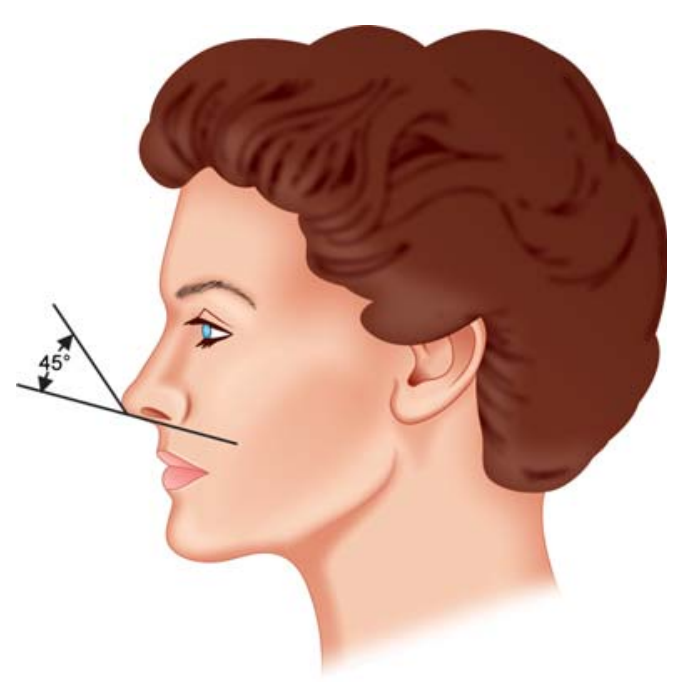

Fig. 29: Assessment of the columella-lobular angle

there is increased fullness here, which is normally caused by a prominent caudal septum, there will be an appearance of increased tip rotation even if the nasolabial angle is, in fact, correct. Finally the columella-lobular angle should be determined (Fig. 29). This should be approximately $45^{\circ}$.

After this assessment the interior of the nose is examined before and after decongestion if necessary. This can best be done initially by tilting the tip and simple inspection with a light source such as a torch in the first instance. Tip recoil will give some measure of the amount of tip support and is a useful measure if support has initially appeared to be compromised. Simple inspection of the nose during quiet breathing and then following a forced inspiratory movement will also allow some impression of any valve collapse, and the level of this can then be used by simple insertion to the nose of a Jobson Horne probe or other object that will support the structures at different levels while inspiration is repeated (an augmented Cottle's test). Finally, the septum, turbinates and internal valve area are inspected with a 30 telescope.

Photographs of the nose should always be available to document the initial findings. Good quality color prints form an essential part of the patient record and should include one frontal, two oblique and two lateral views with a basal view as standard. A skyline view is also often helpful. These photographs should be discussed with patient and a plan of what can, and what cannot, be achieved documented fully after discussion. A list of the goals to be achieved should be written in the records and, if no consensus is apparent, and a game plan is not agreed or the patient's desires are unrealistic, then surgery should not proceed and the patient should be referred elsewhere.

\section{REFERENCES}

1. Daniel RK, Letorneau A. Rhinoplasty: Nasal anatomy. Ann Plast Surg 1987;20(1):5.

2. Bruintjes TD, van Olphen AF, Hillen B. Review of the functional anatomy of the cartilages and muscles of the nose. Rhinology. 1996 Jun;34(2):66-74.

3. Sheen JH, Sheen A. Aesthetic rhinoplasty (2nd ed). Fundamentals and techniques. St Louis, Missouri: Quality Medical Publishing Inc 1998;1:3-57.

4. Marshall AH, Johnston MN, Jones NS. The principles of correcting the septum in septorhinoplasty: Two point fixation. J Laryngol and Otol 1999;113(5):405-12.

\section{ABOUT THE AUTHOR}

\section{Guy Kenyon}

Consultant, Department of Otolaryngology, Whipps Cross University Hospital, London, United Kingdom, e-mail: guykenyon@aol.com 